\begin{tabular}{|c|l|}
\hline Title & Photothermal probing of inhomogeneously modulated transparent thin films \\
\hline Author(s) & Wright, O. B.; Li V oti, R.; Matsuda, O.; Larciprete, M. C.; Bertolotti, M.; Sibilia, C. \\
\hline Citation & $\begin{array}{l}\text { JOURNA L OF A PPLIED PHY SICS, 91(8), 5002-5009 } \\
\text { https://doi.org/10.1063/1.1462414 }\end{array}$ \\
\hline Issue Date & 2002-0415 \\
\hline Doc URL & http://hdl.handle.net/2115/5796 \\
\hline Rights & Copyright $\odot 2002$ A merican Institute of Physics \\
\hline Type & article \\
\hline File Information & JAP91-8.pdf \\
\hline
\end{tabular}

Instructions for use 


\title{
Photothermal probing of inhomogeneously modulated transparent thin films
}

\author{
O. B. Wright ${ }^{a)}$ \\ Department of Applied Physics, Faculty of Engineering, Hokkaido University, Sapporo 060-8628, Japan \\ R. Li Voti \\ INFM and Dipartimento di Energetica, Università degli Studi di Roma "La Sapienza," Via A. Scarpa 16, \\ 00161, Roma, Italy \\ O. Matsuda \\ Department of Applied Physics, Faculty of Engineering, Hokkaido University, Sapporo 060-8628, Japan \\ M. C. Larciprete, C. Sibilia, and M. Bertolotti \\ INFM and Dipartimento di Energetica, Università degli Studi di Roma "La Sapienza," Via A. Scarpa 16, \\ 00161, Roma, Italy
}

(Received 26 November 2001; accepted for publication 29 January 2002)

\begin{abstract}
The problem of the photothermal modulation of optical beams passing through multilayer films is an extremely complex one owing to the inhomogeneously modulated refractive index combined with multiple optical reflections inside the sample. This problem has so far not been given an exact analytical treatment in the field of photothermal probing. We consider here such a treatment for normal-incidence optical probing in reflectance of photothermally modulated single-layer thin-film samples with arbitrary optical constants. The validity of the method is demonstrated by application to a thin transparent film of silica on a silicon substrate. (C) 2002 American Institute of Physics.

[DOI: $10.1063 / 1.1462414]$
\end{abstract}

\section{INTRODUCTION}

Photothermal probing of thin films subject to periodic optical excitation is a well-established method for the determination of film thickness and thermal properties, and has lead to numerous practical applications in nondestructive testing. The thin film under investigation is illuminated with a chopped optical excitation beam, focused in general to a small (typically, sub-100 $\mu \mathrm{m}$ ) spot on the surface, and this induces thermal waves that penetrate into the sample. The resulting near-surface temperature oscillations or surface vibrations, both related to the thermal wave propagation, can be measured using a probe laser beam through optical reflectance variations or beam deflection changes. ${ }^{1-4}$ In particular, the use of normal-incidence probe beams is convenient for samples with an optically smooth surface because it allows the probe beam to be finely focused to a small measurement spot on the surface, and unlike surface-skimming optical probe configurations, can be applied to samples with a gentle concave curvature. By varying the chopping frequency $f$ of the excitation beam the thermal diffusion depth $\mu$, proportional to $1 / f^{1 / 2}$, can be varied, and by fitting to the frequency dependence of the signal in the region in which $\mu \sim d$ ( $d$ the film thickness) the thermal properties or thickness of the thin film can be derived. This method is straightforward when the thin film is opaque to both the exciting and probing radiation.

If, however, the thin film is transparent or partially transparent to the probing radiation the problem becomes more

\footnotetext{
${ }^{a)}$ Electronic mail: assp@kino-ap.eng.hokudai.ac.jp
}

difficult. For example, in the case of a transparent film on an opaque substrate, the optical interaction with the sample is complicated by multiple reflections in the film. Moreover, the modulation of the probe beam by the inhomogeneous temperature distribution cannot be approximated to a surface absorption problem as in the case of opaque films. Rather, the probe beam effectively samples the temperature-induced changes in refractive index at a distribution of depths within the sample simultaneously. The complexity of the analysis has impeded research in this field. Numerical calculations of such effects in thin transparent films using beam deflection methods have been reported, ${ }^{2}$ and approximate analytical calculations based on average temperatures within transparent films have been proposed for reflectance detection methods. ${ }^{5}$ But, no simple and rigorous analytical method has been proposed for application in this field. In this article we show how a general analytical method can be used to deal with reflectance modulation in photothermal experiments in transparent thin films with normal-incidence optical detection methods, ${ }^{6,7}$ and demonstrate the method by application to a thin film of silica on a silicon substrate.

\section{EXPERIMENT AND RESULTS FOR A TRANSPARENT FILM}

The apparatus used is that of a standard modulated photothermal reflectance set-up. Light from an Ar-ion laser at wavelength $488 \mathrm{~nm}$ is chopped by an acousto-optic modulator (AOM) and focused to an approximately Gaussian spot of precisely measured size $90 \mu \mathrm{m}\left(1 / e^{2}\right.$ intensity radius) on the sample at an angle of $16^{\circ}$ with $s$ polarization. The average 


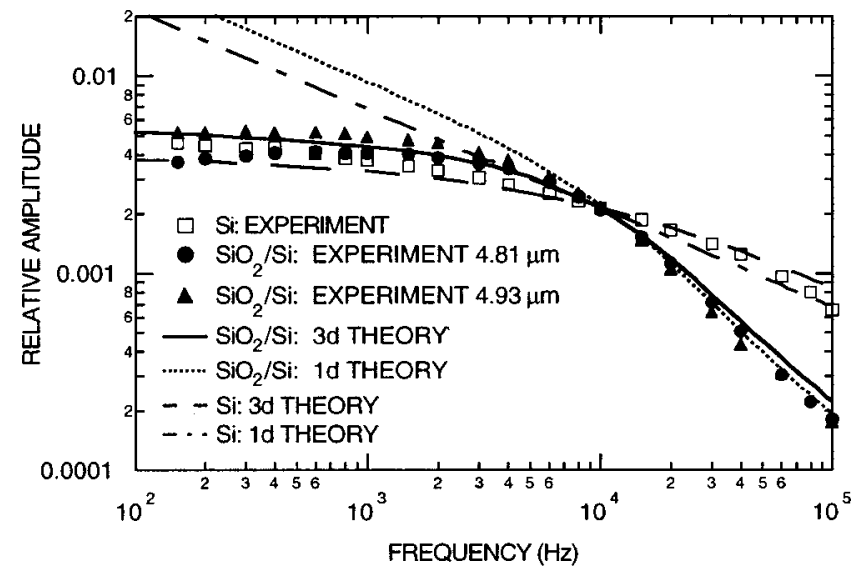

FIG. 1. Plot of the relative amplitude of the reflectance modulation as a function of chopping frequency. The closed circles represent data for a silica film of thickness $4.81 \mu \mathrm{m}$ on a silicon substrate. (The vertical axis corresponds to these data.) The closed triangles represent normalized data for a region of thickness $4.93 \mu \mathrm{m}$ of the same film. The open squares represent the normalized data for a sample of silicon. Dotted line: $1 d$ theory for the silica film system. Solid line: 3d theory for the silica film system. Dasheddotted line: $1 d$ theory for the silicon sample. Dashed line: $3 d$ theory for the silicon sample. The curves are normalized to the same value at a chopping frequency of $10 \mathrm{kHz}$

incident power before the sample was about $200 \mathrm{~mW}$. In the samples used this is expected to produce a typical ac temperature excursions of $\sim 5 \mathrm{~K}$. A HeNe laser beam of wavelength $633 \mathrm{~nm}$ was passed through a nonpolarizing beam splitter (NPBS) and focused at normal incidence to the same point on the sample with a spot size of $30 \mu \mathrm{m}$ and incident optical power $\sim 2 \mathrm{~mW}$. After reflection from the sample, the $\mathrm{HeNe}$ beam is reflected by the NPBS onto one side of a dual-cell photodiode. The other side of the photodiode is illuminated by part of the $\mathrm{HeNe}$ beam derived from the beam before reflection from the sample for purposes of intensity noise reduction. The frequency of the AOM is scanned from $200 \mathrm{~Hz}$ to $100 \mathrm{kHz}$, and by means of lock-in detection the modulated reflectance signal amplitude and phase are recorded as a function of frequency. Reference scans with the chopped and attenuated Ar laser beam focused directly on the photodiode are used to correct for the instrumental frequency response. A sample of vitreous silica of nominal thickness $5 \mu \mathrm{m}$ was prepared by thermal oxidation on a (100) silicon wafer substrate of thickness $500 \mu \mathrm{m}$. A reference bare (100) silicon wafer was also used for comparison.

The closed circles in Figs. 1 and 2 show the measured relative amplitude and phase variations for the case in which the optical beams are incident on the central portion of the silica film sample. The amplitude decreases as the frequency increases, whereas the phase lag with respect to the periodic optical excitation increases. Spectroscopic ellipsometry measurements at the same point on the sample allowed a precise measurement of the film thickness, $4.81 \pm 0.01 \mu \mathrm{m}$, at this point. The closed triangles in Figs. 1 and 2 correspond to measurements taken at a point on the sample distant $\sim 2 \mathrm{~cm}$ from the first point, at which position ellipsometry measurements give a thickness of $4.93 \pm 0.01 \mu \mathrm{m}$. The amplitude variation is very similar to that for the $4.81 \mu \mathrm{m}$ point, whereas the phase variation is changed by approximately

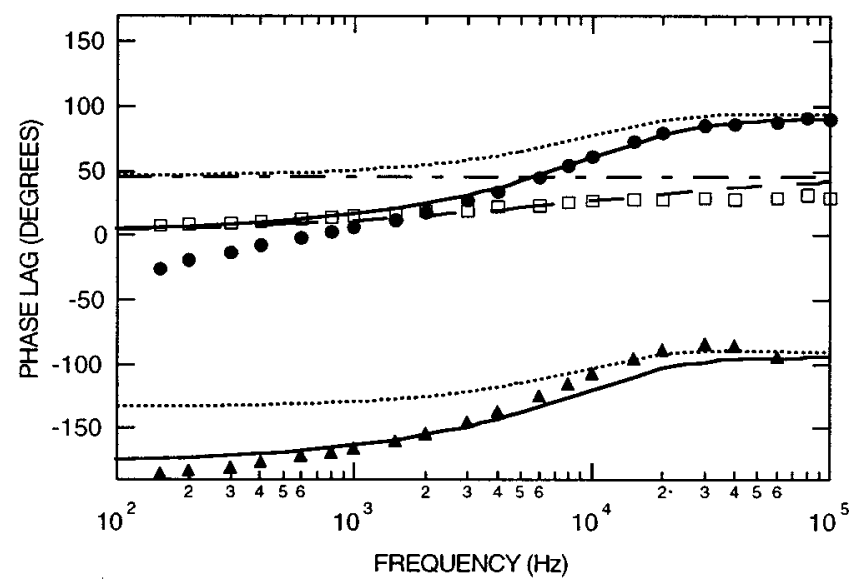

FIG. 2. Plot of the photothermal phase lag as a function of chopping frequency. The closed circles represent data for a silica film of thickness 4.81 $\mu \mathrm{m}$ on a silicon substrate. The closed triangles represent data for a region of thickness $4.93 \mu \mathrm{m}$ of the same film. The open squares represent the data for a sample of silicon. Upper dotted line: $1 d$ theory for the silica film of thickness $4.81 \mu \mathrm{m}$. Lower dotted line: $1 d$ theory for the silica film of thickness $4.93 \mu \mathrm{m}$. Upper solid line: $3 d$ theory for the silica film of thickness $4.81 \mu \mathrm{m}$. Lower solid line: $3 d$ theory for the silica film of thickness 4.93 $\mu \mathrm{m}$. Dashed-dotted line: $1 d$ theory for the silicon sample. Dashed line: $3 d$ theory for the silicon sample.

$180^{\circ}$ with respect to the corresponding variation for 4.81 $\mu \mathrm{m}$. Such a phase inversion phenomenon is expected in transparent sample systems because of a periodicity in sample reflectivity at the probe wavelength as a function of thickness. ${ }^{5}$ Also shown in Figs. 1 and 2 by the open-square symbols are the results for the sample of bulk silicon. These results for silicon will be discussed after those of silica.

\section{COMPARISON WITH THE THEORY FOR A TRANSPARENT FILM}

In order to model these data for the silica film we must take into account the inhomogeneous temperature distribution in the sample. In the present silica thin film the thermal diffusion length is of the order of or smaller than the film thickness at a frequencies above $\sim 10 \mathrm{kHz}$, at which frequencies the temperature distribution in the film becomes particularly inhomogeneous and, moreover, there exists a significant thermal wave phase variation across the film. The problem of reflectance modulation in a single ${ }^{6,7}$ or multilayer $^{7}$ film system subject to a small inhomogeneous perturbation in refractive index and strain has been considered in the context of laser picosecond acoustics. Both problems involving light reflectance as well as light transmission have been considered, but we shall be working only in reflectance and with a single transparent layer in the present study. For a single film of thickness $d$ of arbitrary dielectric constant $\epsilon_{1}$ on a substrate of dielectric constant $\epsilon_{2}$ subject to a small spatially dependent perturbation in the normal direction $\Delta \epsilon(z)$, the variation $\delta r$ in the complex amplitude reflectance $r$ of such a system is given by 


$$
\begin{aligned}
\frac{\delta r(t)}{r}= & \frac{i k}{2 a_{0} b_{0}}\left\{\int_{0}^{d} \Delta \epsilon\left(z^{\prime}\right)\left(a_{1} e^{i k_{1} z^{\prime}}+b_{1} e^{-i k_{1} z^{\prime}}\right)^{2} d z^{\prime}\right. \\
& +\int_{d}^{\infty} \Delta \epsilon\left(z^{\prime}\right) a_{2}^{2} e^{2 i k_{2}\left(z^{\prime}-d\right)} d z^{\prime}+\left(a_{1}+b_{1}\right)^{2} \\
& \left.\times\left(1-\epsilon_{1}\right) u(0)+a_{2}^{2}\left(\epsilon_{1}-\epsilon_{2}\right) u(d)\right\},
\end{aligned}
$$

with

$$
\begin{aligned}
& a_{0}=\left(k+k_{1}\right)\left(k_{1}+k_{2}\right)+\left(k-k_{1}\right)\left(k_{1}-k_{2}\right) e^{2 i k_{1} d}, \\
& b_{0}=\left(k-k_{1}\right)\left(k_{1}+k_{2}\right)+\left(k+k_{1}\right)\left(k_{1}-k_{2}\right) e^{2 i k_{1} d}, \\
& a_{1}=2 k\left(k_{1}+k_{2}\right), \\
& b_{1}=2 k\left(k_{1}-k_{2}\right) e^{2 i k_{1} d}, \\
& a_{2}=4 k k_{1} e^{i k_{1} d} .
\end{aligned}
$$

Here, $k=2 \pi / \lambda$ is the wave number at the probe wavelength in vacuum, $k_{1}=\sqrt{\epsilon_{1}} k$ is that in the film, $k_{2}=\sqrt{\epsilon_{2}} k$ is that in the substrate, $u(0)$ is the inward displacement of the film top surface, and $u(d)$ the inward displacement of the filmsubstrate interface. The first two terms in the curly brackets represent the contribution to $\delta r / r$ from the variation in the dielectric constant of the film and substrate, respectively, whereas the last two terms represent the contribution to $\delta r / r$ from the motion of the sample surface and interface (arising through interference effects in the film). In the present experiment the thermal waves lead in general to contributions from all four terms because of the coupling of the dielectric constant (and, therefore, the refractive index) with temperature and because of the thermal expansion of the film and substrate. In Eq. (1) the complex notation involving $i$ refers to the variation at the periodicity of the optical wave. In contrast, the chopping frequency affects the temporal variation $\delta r(t) / r$ through the spatial and temporal variation (periodic in $1 / f)$ of the functions $\Delta \epsilon(z)=\Delta \epsilon(z, t), u(0)$ $=u(0, t)$, and $u(d)=u(d, t)$. Since the period of the optical chopping in the present experiment is very small compared to the characteristic time for acoustic propagation out of the heated region, the displacements can be evaluated from

$$
\begin{aligned}
& u(d)=-\alpha_{2} \int_{d}^{\infty} T(z, t) d z, \\
& u(0)=-\alpha_{1} \int_{0}^{d} T(z, t) d z+u(d),
\end{aligned}
$$

where $\alpha_{1}$ and $\alpha_{2}$ are the linear coefficients of thermal expansion of the film and substrate, respectively, and $T(z, t)$ is the temperature deviation from thermal equilibrium. The displacement $u$ is negative for an outward expansion. The dielectric constant change is also related to the temperature distribution through

$$
\Delta \epsilon(z, t)=\frac{d \epsilon}{d T} T(z, t)=2(N+i K)\left(\frac{d N}{d T}+i \frac{d K}{d T}\right) T(z, t),
$$

where $N+i K=\sqrt{\epsilon}$ is the probe-wavelength refractive index (that we shall refer to as $N_{1}+i K_{1}$ for the film and $N_{2}$ $+i K_{2}$ for the substrate). The relative reflectivity variation measured in experiment is given by $\delta R / R=2 \operatorname{Re}(\delta r / r)$. Unlike previous treatments for analysis of the reflectivity of transparent films in photothermal experiments, Eq. (1) fully takes into account the effect of the distributed perturbation in refractive index of the sample and all multiple optical reflections therein.

In order to determine the reflectivity variation theoretically one must first evaluate the periodically varying temperature distribution and, subsequently, the perturbation in dielectric constants and the interface motion of the sample. The simplest approach is a one-dimensional (1D) treatment. Although the pump optical beam is absorbed within a finite depth in the silicon substrate (of the order of the optical absorption depth $\sim 0.5 \mu \mathrm{m}$ for the $488 \mathrm{~nm}$ excitation beam), this distance is smaller than any other characteristic distance in the experiment (the film thickness or the thermal diffusion lengths), and so it is a reasonable first approximation to solve the thermal wave problem assuming surface absorption at the silicon substrate. The absorbed light intensity variation is taken as $\left(I_{0} / 2\right)[1+\exp (-i \omega t)]$, where $\omega=2 \pi f$. Then, the temperature variation in the sample according to the theory of one-dimensional Fourier heat conduction is given by

$$
\begin{aligned}
& T(z, t)=\frac{I_{0} \exp (-i \omega t)}{2\left(e_{1}+e_{2}\right) \sqrt{-i \omega}} \frac{\exp \left[\sqrt{-2 i}(z-d) / \mu_{1}\right]+\exp \left[-\sqrt{-2 i}(z+d) / \mu_{1}\right]}{1-R_{1 \mathrm{D}} \exp \left[-\sqrt{-2 i} 2 d / \mu_{1}\right]} \quad 0 \leqslant z \leqslant d, \\
& T(z, t)=\frac{I_{0} \exp (-i \omega t)}{2\left(e_{1}+e_{2}\right) \sqrt{-i \omega}} \frac{\left(1+\exp \left[-\sqrt{-2 i} 2 d / \mu_{1}\right]\right) \exp \left[-\sqrt{-2 i}(z-d) / \mu_{2}\right]}{1-R_{1 \mathrm{D}} \exp \left[-\sqrt{-2 i} 2 d / \mu_{1}\right]} \quad z \geqslant d,
\end{aligned}
$$


TABLE I. Values of physical parameters used. Unless otherwise stated the refractive indices, optical absorption depths, and thermo-optic coefficients refer to the probe wavelength $633 \mathrm{~nm}$. The parameters given correspond to a temperature of $300 \mathrm{~K}$.

\begin{tabular}{|c|c|c|c|c|}
\hline Solid & Parameter & Symbol & Value & Ref./Method \\
\hline $\mathrm{SiO}_{2}$ & Refractive index & $N_{1}+i K_{1}$ & $1.50+0.0026 i$ & Measured by ellipsometry \\
\hline $\mathrm{SiO}_{2}$ & Optical absorption depth & $\zeta_{1}=\lambda / 4 \pi K_{1}$ & $19.4 \mu \mathrm{m}$ & Measured by ellipsometry \\
\hline $\mathrm{SiO}_{2}$ & Thermo-optic coefficient & $d N_{1} / d T$ & $1.2 \times 10^{-3} \mathrm{~K}^{-1}$ & Ref. 8 \\
\hline $\mathrm{SiO}_{2}$ & Thermo-optic coefficient & $d K_{1} / d T$ & 0 & Assumed to be negligible \\
\hline $\mathrm{SiO}_{2}$ & Thermal conductivity & $\kappa_{1}$ & $1.4 \mathrm{~W} \mathrm{~m}^{-1} \mathrm{~K}^{-1}$ & Ref. 9 \\
\hline $\mathrm{SiO}_{2}$ & Specific heat capacity & $c_{1}$ & $760 \mathrm{~J} \mathrm{~kg}^{-1} \mathrm{~K}^{-1}$ & Ref. 10 \\
\hline $\mathrm{SiO}_{2}$ & Density & $\rho_{1}$ & $2200 \mathrm{~kg} \mathrm{~m}^{-3}$ & Ref. 11 \\
\hline $\mathrm{SiO}_{2}$ & Linear thermal expansion coefficient & $\beta_{1}$ & $5.5 \times 10^{-7} \mathrm{~K}^{-1}$ & Ref. 12 \\
\hline $\mathrm{SiO}_{2}$ & Refractive index (at pump $\lambda$ ) & $N_{1}^{\prime}+i K_{1}^{\prime}$ & $1.51+0.0059 i$ & Measured by ellipsometry \\
\hline $\mathrm{SiO}_{2}$ & Thermal diffusion length at $100 \mathrm{~Hz}$ & $\mu_{1}$ & $52 \mu \mathrm{m}$ & $(\kappa / c \rho \pi f)^{1 / 2}$ \\
\hline $\mathrm{SiO}_{2}$ & Thermal diffusion length at $10 \mathrm{kHz}$ & $\mu_{1}$ & $5.2 \mu \mathrm{m}$ & $(\kappa / c \rho \pi f)^{1 / 2}$ \\
\hline $\mathrm{SiO}_{2}$ & Thermal diffusion length at $100 \mathrm{kHz}$ & $\mu_{1}$ & $1.6 \mu \mathrm{m}$ & $(\kappa / c \rho \pi f)^{1 / 2}$ \\
\hline $\mathrm{Si}$ & Refractive index & $N_{2}+i K_{2}$ & $3.875+0.0211 i$ & Measured by ellipsometry \\
\hline $\mathrm{Si}$ & Optical absorption depth & $\zeta_{2}=\lambda / 4 \pi K_{1}$ & $2.4 \mu \mathrm{m}$ & Measured by ellipsometry \\
\hline $\mathrm{Si}$ & Thermo-optic coefficient & $d N_{2} / d T$ & $3.3 \times 10^{-4} \mathrm{~K}^{-1}$ & Ref. 8 \\
\hline $\mathrm{Si}$ & Thermo-optic coefficient & $d K_{2} / d T$ & 0 & Assumed to be negligible \\
\hline $\mathrm{Si}$ & Thermal conductivity & $\kappa_{2}$ & $148 \mathrm{~W} \mathrm{~m}^{-1} \mathrm{~K}^{-1}$ & Ref. 9 \\
\hline $\mathrm{Si}$ & Specific heat capacity & $c_{2}$ & $705 \mathrm{~J} \mathrm{~kg}^{-1} \mathrm{~K}^{-1}$ & Ref. 10 \\
\hline $\mathrm{Si}$ & Density & $\rho_{2}$ & $2330 \mathrm{~kg} \mathrm{~m}^{-3}$ & Ref. 11 \\
\hline $\mathrm{Si}$ & Linear thermal expansion coefficient & $\beta_{2}$ & $2.6 \times 10^{-6} \mathrm{~K}^{-1}$ & Ref. 12 \\
\hline $\mathrm{Si}$ & Refractive index (at pump $\lambda$ ) & $N_{2}^{\prime}+i K_{2}^{\prime}$ & $4.367+0.079 i$ & Ref. 8 \\
\hline $\mathrm{Si}$ & Thermal diffusion length at $100 \mathrm{~Hz}$ & $\mu_{2}$ & $540 \mu \mathrm{m}$ & $(\kappa / c \rho \pi f)^{1 / 2}$ \\
\hline $\mathrm{Si}$ & Thermal diffusion length at $10 \mathrm{kHz}$ & $\mu_{2}$ & $54 \mu \mathrm{m}$ & $(\kappa / c \rho \pi f)^{1 / 2}$ \\
\hline $\mathrm{Si}$ & Thermal diffusion length at $100 \mathrm{kHz}$ & $\mu_{2}$ & $17 \mu \mathrm{m}$ & $(\kappa / c \rho \pi f)^{1 / 2}$ \\
\hline
\end{tabular}

where $R_{1 \mathrm{D}}=\left(e_{1}-e_{2}\right) /\left(e_{1}+e_{2}\right)$ is the thermal wave reflection coefficient at the interface, $e$ is the thermal effusivity $(=\sqrt{\kappa c \rho}, \kappa$ the thermal conductivity, $c$ the specific heat, and $\rho$ the density), $\mu=(2 D / \omega)^{1 / 2}$ is the thermal diffusion length ( $D=\kappa / c \rho$ the thermal diffusivity), and where the subscripts 1 and 2 refer to the film and substrate, respectively. [The complex notation involving $i$ in Eq. (5) refers to the modulation at the chopping frequency $f$, and does not refer to the optical fre-quency.] For ease of calculation it is pertinent to consider the relative magnitude of the various terms in Eq. (1) for the present experiment. Parameters important to the problem at hand are listed in Table $\mathrm{I}^{8-12}$ In particular, the value of $d N / d T$ for the silica film $\left(d N_{1} / d T=1.2\right.$ $\left.\times 10^{-3} \mathrm{~K}^{-1}\right)$ is significantly greater than that of the silicon substrate

$\left(d N_{2} / d T=3.3 \times 10^{-4} \mathrm{~K}^{-1}\right)$. Moreover, the effect of multiple reflection of light inside the transparent silica film tends to greatly enhance the photothermal coupling of light with the film material. ${ }^{5}$ The result is that the magnitude of the contribution of the second integral in Eq. (1) is usually very small compared to that of the first $\left(\sim 10^{-3}\right.$ times $)$. Moreover, the terms arising from thermal expansion, the third and fourth terms in Eq. (1), are also usually very small compared to the contribution from the first $\left(\sim 10^{-3}\right.$ times $)$. Under the present experimental conditions only the first term in Eq. (1) is important for analyzing our results. Because of this, only the first term in Eq. (1) is included in the analysis for the moment. Conditions and predictions for the case in which the first term in Eq. (1) can become small are discussed later in this article.

The dotted curve in Fig. 1 corresponds to the calculation of the expected reflectance amplitude variation with chopping frequency for $d=4.81 \mu \mathrm{m}$ using Eqs. (1)-(4), the (real) temperature variation of Eq. (5), and the parameters of Table I. The theoretical curve is normalized to the same value as the experiment at $10 \mathrm{kHz}$ for convenience of comparison. At 
high frequencies the agreement between theory and experiment (closed circles) is excellent, but at lower frequencies there is a discrepancy. The deviation from experiment is even more pronounced in the photothermal phase lag, as shown in Fig. 2 by the upper dotted curve (theory) and the experimental points (closed circles).

These results suggest that a three-dimensional (3D) treatment of the heat conduction problem would be more appropriate, particularly at lower frequencies for which the thermal diffusion length in the film or substrate (see Table I) becomes larger than the optical spot radius of the excitation beam. For a Gaussian excitation beam of radius $a$ (for $1 / e^{2}$ intensity) modulated sinusoidally in time to give an average absorbed power $P$ in the sample, ${ }^{2,5,13}$

$$
\begin{aligned}
T(r, z, t) & =\frac{1}{2 \pi} \int_{0}^{\infty} \widetilde{T}(p, z, t) J_{0}(p r) p d p \\
& =\frac{1}{2 \pi} \int_{0}^{\infty} A(p, z, t) \exp \left[-\frac{(p a)^{2}}{8}\right] J_{0}(p r) p d p,
\end{aligned}
$$

where $\widetilde{T}$ is the Hankel transformation of the temperature in the domain $p$, and $J_{0}$ is the zero-order Bessel function. The quantity $\widetilde{T}$ is the product of two terms: the Gaussian term, which is the Hankel transformation of the pump beam shape, and the function $A$ given by

$$
A(p, z, t)=\frac{P \exp (-i \omega t)}{\left(\kappa_{1} \sqrt{p^{2}-\left(2 i / \mu_{1}^{2}\right)}+\kappa_{2} \sqrt{p^{2}-\left(2 i / \mu_{2}^{2}\right)}\right)} \frac{\exp \left[(z-d) \sqrt{p^{2}-\left(2 i / \mu_{1}^{2}\right)}\right]+\exp \left[-(z+d) \sqrt{p^{2}-\left(2 i / \mu_{1}^{2}\right)}\right]}{1-R_{3 \mathrm{D}} \exp \left[-2 d \sqrt{p^{2}-\left(2 i / \mu_{1}^{2}\right)}\right]},
$$

where

$$
R_{3 \mathrm{D}}=\frac{\kappa_{1} \sqrt{p^{2}-\left(2 i / \mu_{1}^{2}\right)}-\kappa_{2} \sqrt{p^{2}-\left(2 i / \mu_{2}^{2}\right)}}{\kappa_{1} \sqrt{p^{2}-\left(2 i / \mu_{1}^{2}\right)}+\kappa_{2} \sqrt{p^{2}-\left(2 i / \mu_{2}^{2}\right)}} .
$$

Equation (6b) applies for $0 \leqslant z \leqslant d$. As in Eq. (5) a similar equation applies for $z \geqslant d$ (not shown here). When $p \rightarrow 0$, $R_{3 \mathrm{D}} \rightarrow R_{1 \mathrm{D}}$, and Eq. (6b) tends to the temperature for the onedimensional case in Eq. (5), as expected. (This can be done using the substitution $P=\pi a^{2} I_{0} / 4$, where $I_{0}$ is the maximum absorbed optical intensity at the spot center during the chopping cycle.) In the present experiment the radius of the probe beam is three times smaller than that of the excitation beam, and so the values of $T(r, z, t)$ at $r=0$ in Eqs. (6) are appropriate for use in Eqs. (2)-(4). Combining Eqs. (6) at $r=0$ with Eq. (1) and using the known value of $a=90 \mu \mathrm{m}$, the solid theoretical curve in Fig. 1 is obtained. The agreement with experiment is reasonable over the whole range of the frequency spectrum studied. The agreement with the phase lag of the optical reflectance signal in Fig. 2 is similarly improved, although a residual discrepancy at the lowest frequencies remains. (Note that we have neglected the effect of the finite substrate thickness $\sim \mu_{2}$ at $200 \mathrm{~Hz}$-see Table I.) The theoretical fitting is done without introducing any variable parameters, with constants taken only from the literature or from ellipsometry measurements. The neglect of the finite radius of the probe $\mathrm{HeNe}$ laser beam is justified in the present case. In fact, it can be shown that Eqs. (6) with $r=0$ still apply for a general probe beam radius $b$ if the quantity $a$ is replaced by $\left(a^{2}+b^{2}\right)^{1 / 2}$ (see the Appendix). In the present case this represents a negligible correction for the chosen value of $b=30 \mu \mathrm{m}$.

The phase of the photothermal signal in transparent films is known to show inversions due to the periodic dependence of the reflectivity on film thickness. ${ }^{2,5}$ In order to test whether this effect could be experimentally observed for the present sample and theoretically reproduced, we measured a point on the film distant by $\sim 2 \mathrm{~cm}$ from the first point. Ellipsometry measurements gave a film thickness of 4.93 $\pm 0.01 \mu \mathrm{m}$ at this second point. The corresponding photothermal amplitude and phase lag variations are shown by the solid triangles in Figs. 1 and 2. The photothermal amplitude is very similar to that for $d=4.81 \mu \mathrm{m}$. In contrast, the phase variation is shifted by approximately $180^{\circ}$. Comparison with the theory based on the three-dimensional heat diffusion equation again gives good agreement with the experimental results. The predicted curve for amplitude lies essentially on top of that for $4.81 \mu \mathrm{m}$ and so is not shown separately, whereas that for the phase lag is shown by the lower solid curve in Fig. 2. As with the results for $d=4.81 \mu \mathrm{m}$, the agreement for this theory is much better than that for the one-dimensional treatment of heat diffusion (lower dotted curve). The discrepancy at lower frequencies is caused by the thermal diffusion length in the substrate becoming greater than the optical spot size, producing a temperature distribution no longer well approximated by a one-dimensional treatment. The two theories agree at higher frequencies, as expected. ${ }^{14}$

\section{RESULTS AND THEORY FOR A BULK SAMPLE}

The results for the bulk sample of silicon (100) (of thickness $250 \mu \mathrm{m}$ ), also shown in Figs. 1 and 2, provide a check on the experimental technique. A one-dimensional treatment of the heat diffusion problem in an opaque material with surface periodic heating leads to a $1 / f^{1 / 2}$ dependence of the amplitude on frequency, as shown by the dashed-dotted curve in Fig. 1. ${ }^{15}$ Again, this model is only adequate in the high-frequency regime. The phase lag is predicted to take a value of $45^{\circ},{ }^{15}$ only in approximate agreement with experiment near the top of the frequency range. As before, however, the three-dimensional treatment of the problem (dashed curves) gives good agreement with the experimental amplitude and phase lag curves for silicon (open-square symbols) 


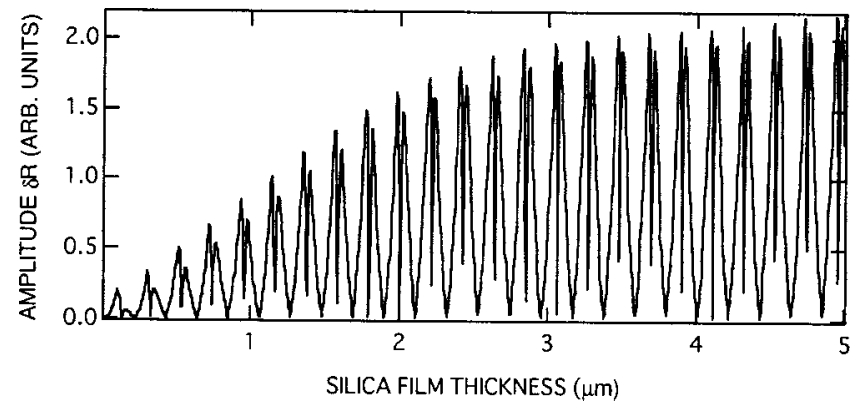

FIG. 3. Plot of the relative amplitude of the reflectance modulation as a function of film thickness for a silica film on silicon with the same optical and thermal properties as the present samples. The reflectance variation of the pump optical beam is not included in this plot.

over the whole frequency range covered. The variations over the whole frequency range are less pronounced than for the silica film sample because the latter sample involves a large variation with frequency of the thermal wave penetration in the transparent portion of the sample.

\section{DISCUSSION AND CONCLUSIONS}

In order to test the linearity of the present measurements we carried out measurements at fluences $\sim 7$ times smaller than those used above. The results were in reasonable agreement with those above, indicating that static heating effects in the samples, due for example to their finite thicknesses, were not producing significant nonlinearities.

It is interesting to map out the response $\delta R$ of the present sample as a function of film thickness to see what effect this has on the signal in general. Figures 3 and 4 show the predicted amplitude and phase lag variations of the reflectivity as a function of film thickness at $10 \mathrm{kHz}$ for film thicknesses ranging from 0 to $5 \mu \mathrm{m}$ using the one-dimensional model for heat conduction (a reasonable approximation at this frequency). All four contributions in Eq. (1) are included here. The variation of the optical pump reflectance with thickness has been excluded from this plot to make clearer the effects of the probe. Both amplitude and phase show periodic variations with thickness. The points for which the amplitude becomes small correspond to points near to those for which $\partial R / \partial d=0$ ( $d$, the film thickness), that is, to maxima and minima in the probe reflectivity $(R)$ variation as a function of $d$. It is at these points that the phase inversions occur because $\partial R / \partial d$ changes sign. Because of this periodicity it is clear that single-frequency photothermal measurements would not allow a unique determination of film thickness, and that the frequency scan method as used here is, therefore, essential. From Fig. 4 it is evident that in the region of the phase inversions there are spikes in phase. These spikes arise because in the region of these transitions the film photothermal coupling contribution $\left(\propto d N_{1} / d T\right)$ becomes smaller than or of the same order as the substrate photothermal coupling contribution $\left[\propto d N_{2} / d T\right.$ - the second term in Eq. (1)] or the thermal expansion contribution [the third and fourth terms in Eq. (1)]. These substrate photothermal coupling and thermal

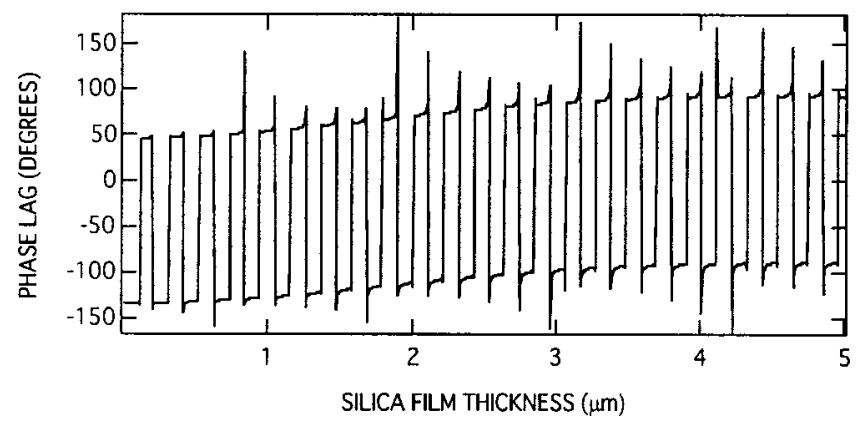

FIG. 4. Plot of the photothermal phase lag as function of film thickness for a silica film on silicon with the same optical and thermal properties as the present samples.

expansion contributions have a different phase behavior from the film photothermal coupling contribution, leading to the spikes in Fig. 4.

The inclusion of the dependence of absorption of the pump laser beam on thickness would slightly alter the curves in Figs. 3 and 4. For the present sample the periodic variation caused by pump beam multiple reflections is typically only $\sim \pm 30 \%$ in the region in which the sample thickness is $\sim 5$ $\mu \mathrm{m}$. The maxima and minima of this variation depend on thickness because of the finite optical absorption of the silica film. In fact, we have completely neglected the effect of this absorption in the thermal wave generation process, although we have accounted for it in the detection process through Eq. (1). A more sophisticated treatment of the generation process is at present under study.

In conclusion, we have presented a method that can treat the general problem of modulated reflectance from inhomogeneous variations of refractive indices in a transparent film on an opaque substrate. The present article has dealt with the simplest case of a single transparent layer, and good agreement is obtained between experiment and theory. However, analytical techniques similar to the one presented here can equally well be applied to photothermal reflectance modulation in an arbitrary multilayer stack of transparent or partially transparent materials. Moreover, it is possible to work in transmission as well as in reflectance. Our approach should therefore lead to better methods for quantitative analysis of the thermal properties and thicknesses of multilayer samples and to new applications in nondestructive testing.

\section{ACKNOWLEDGMENT}

The authors are very grateful to A. Matthew for supplying the silica sample.

\section{APPENDIX}

In this Appendix we consider the effect of the probe beam radius $b$ on the reflectance variation. Equation (6) allows the calculation of the temperature rise when the sample is heated by a Gaussian pump beam of radius $a$ (for $1 / e^{2}$ intensity) chopped at the frequency $\omega / 2 \pi$. When a probe ray is sent parallel to the pump beam with a pump-probe offset $r_{0}$ (the distance between the pump beam axis and the probe ray), the reflectance variation calculated from Eq. (4) and the 

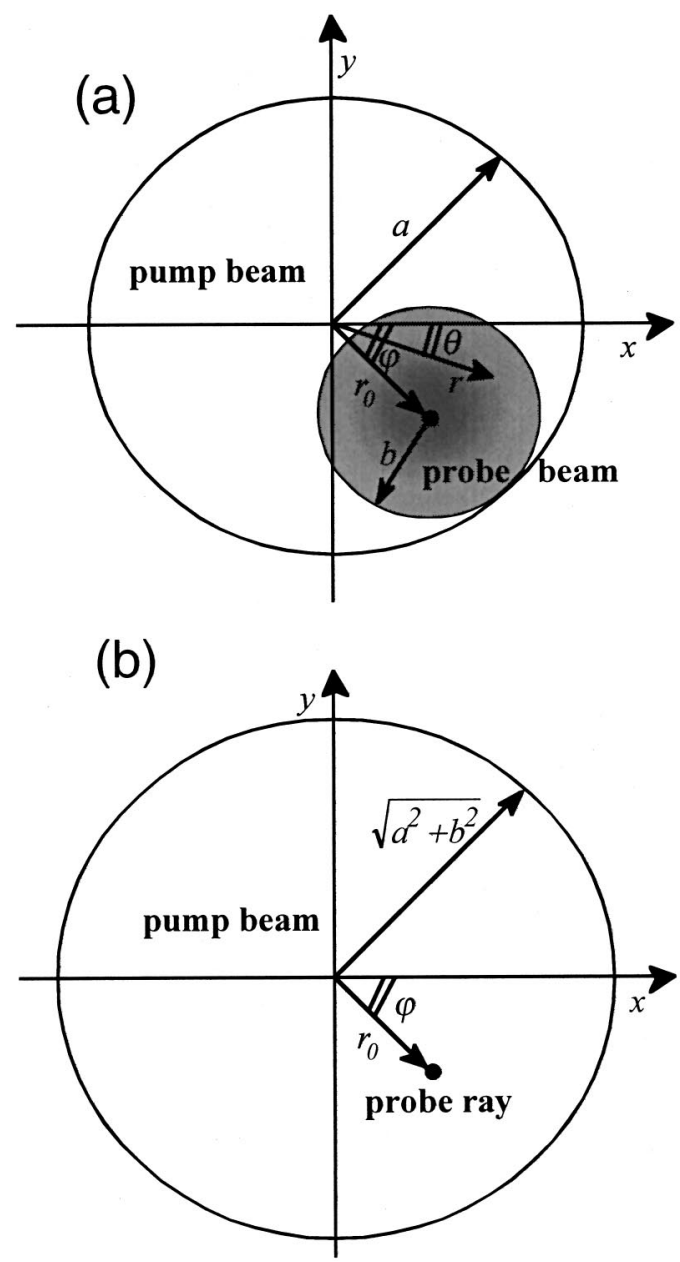

FIG. 5. Diagram to show the equivalence (in terms of the reflectance variation) between a finite radius probe beam system and an infinitely thin probe beam (probe ray) system.

first term in Eq. (1) depends only on the temperature rise $T\left(r_{0}, z\right)$ from Eq. (6) corresponding to the position of the probe ray.

In practice the Gaussian probe beam will have a finite radius $b$ [see Fig. 5(a)]. In this case, we may consider the probe beam as made up of many rays, calculate the reflectance variation for each ray, and finally integrate all these contributions over the whole probe beam profile. ${ }^{16}$ As a result, the total reflectance variation, through the linear theory of Eq. (1), should depend on the temperature rise $T_{\mathrm{av}}$ $=T_{\mathrm{av}}(z)$ averaged over the probe beam intensity profile $\mathrm{w}$ as follows:

$$
T_{\mathrm{av}}=\frac{\iint T(x, y, z) w(x, y) d x d y}{\iint w(x, y) d x d y},
$$

where the Gaussian intensity profile of the probe is given by

$$
w=w_{0} \exp \left[-2 \frac{\left(x-x_{0}\right)^{2}+\left(y-y_{0}\right)^{2}}{b^{2}}\right],
$$

and $w_{0}$ is the intensity at the center $C \equiv\left(x_{0}, y_{0}\right)$. For generality we consider $x_{0}$ and $y_{0}$ to be arbitrary here, although in experiment we choose $x_{0}=y_{0}=0$. In cylindrical coordinates one may use $d x d y=r d r d \theta$ and $\left(x-x_{0}\right)^{2}+\left(y-y_{0}\right)^{2}=r^{2}$ $+r_{0}^{2}-2 r r_{0} \cos (\theta-\phi)$, so that Eq. (A1) becomes

$$
\begin{aligned}
T_{\mathrm{av}}= & \frac{2}{\pi b^{2}} \int_{0}^{\infty} T(r, z) \exp \left[-2 \frac{r^{2}+r_{0}^{2}}{b^{2}}\right] r d r \\
& \times \int_{0}^{2 \pi} \exp \left[4 \frac{r r_{0}}{b^{2}} \cos (\theta-\phi)\right] d \theta .
\end{aligned}
$$

Since the second integral in Eq. (A2) gives $2 \pi I_{0}\left(4 r r_{0} / b^{2}\right),{ }^{17}$ where $I_{0}$ is the modified zero-order Bessel function, one may write

$$
T_{\mathrm{av}}=\frac{4}{b^{2}} \int_{0}^{\infty} T(r, z) I_{0}\left(4 \frac{r r_{0}}{b^{2}}\right) \exp \left[-2 \frac{r^{2}+r_{0}^{2}}{b^{2}}\right] r d r .
$$

If the cylindrical symmetry of $T(r, z)$ is considered, then by combining Eqs. (6a) and (A3) one obtains

$$
\begin{aligned}
T_{\mathrm{av}}= & \frac{2}{\pi b^{2}} \int_{0}^{\infty} A(p, z) \exp \left[-\frac{(p a)^{2}}{8}\right] p d p \\
& \times \int_{0}^{\infty} J_{o}(p r) I_{o}\left(4 \frac{r r_{o}}{b^{2}}\right) \exp \left[-2 \frac{r^{2}+r_{o}^{2}}{b^{2}}\right] r d r .
\end{aligned}
$$

Since the second integral in Eq. (A4) gives ${ }^{17}$ $\exp \left[-(p b)^{2} / 8\right] J_{0}\left(p r_{0}\right) b^{2} / 4$, Eq. (A4) finally becomes

$$
T_{\mathrm{av}}=\frac{1}{2 \pi} \int_{0}^{\infty} A(p, z) J_{0}\left(p r_{0}\right) \exp \left[-\frac{p^{2}\left(a^{2}+b^{2}\right)}{8}\right] p d p .
$$

Comparing Eqs. (A5) and (6a), one can see that exactly the same $T_{\text {av }}$ could be obtained by using different pump-probe systems: for example, a Gaussian pump beam with the larger pump radius $\left(a^{2}+b^{2}\right)^{1 / 2}$ combined with a probe ray at the distance $r_{0}$ [see Fig. 5(b)]. In other words, the reflectance variation, which depends on the average response, does not distinguish between thermal excitation (with a Gaussian pump beam) and probe detection (with a Gaussian probe beam); therefore, the same reflectance variation is obtained using any equivalent pump-probe system with the same values of the composite radius $\left(a^{2}+b^{2}\right)^{1 / 2}$ and the pump-probe offset $r_{0}$.

This property may be demonstrated more generally for any causal linear system if it can be described by means of Green's functions (temperature distributions being one example). In fact, if $G$ is the two-dimensional Green's function for an excitation ray (defined by a spatial delta function), and $w_{\text {pump }}$ is the pump beam profile, the temperature rise is given by the convolution $T=G \otimes w_{\text {pump }}$ in the $x-y$ plane. Averaging the temperature over the probe beam profile $w_{\text {probe }}$ corresponds to a second convolution, given by $T_{\mathrm{av}}$ $=T \otimes w_{\text {probe }}=\left(G \otimes w_{\text {pump }}\right) \otimes w_{\text {probe }}=G \otimes\left(w_{\text {pump }} \otimes w_{\text {probe }}\right)=G$ $\otimes w_{\text {pump-probe }}$. If both $w_{\text {pump }}$ and $w_{\text {probe }}$ are two-dimensional Gaussian functions with radii $a$ and $b$, respectively, then $w_{\text {pump-probe }}$ is still a two-dimensional Gaussian function with the equivalent radius $\left(a^{2}+b^{2}\right)^{1 / 2}$, in agreement with the particular case presented above. 
The analysis in this Appendix has been given in terms of averages over the temperature distribution, related to the first two terms in Eq. (1). Since the spatial distribution of the sample thermal expansion is in general different from that of the temperature, the treatment in terms of two Gaussian distributions cannot be applied to the last two terms in Eq. (1). However, the more general analysis in terms of the convolution of the pump and probe beam effects mentioned above can be applied to the last two terms in Eq. (1), in which case $T$ should be replaced by $u(0)$ or $u(d)$, and $w_{\text {pump }}$ should then be interpreted as the lateral profile of the excited surface or interface displacement. A more detailed discussion of this case is not given here because these surface or interface displacement effects are negligible for the data described in the present article.

${ }^{1}$ W. L. Smith, A. Rosencwaig, and D. L. Willenborg, Appl. Phys. Lett. 47, 584 (1985).

${ }^{2}$ J. Opsal, A. Rosencwaig, and D. Willenborg, Appl. Opt. 22, 3169 (1983).

${ }^{3}$ A. Rosencwaig, J. Opsal, W. L. Smith, and D. Willenborg, Appl. Phys. Lett. 46, 1013 (1985).

${ }^{4}$ M. Bertolotti, R. Li Voti, G. Liakhou, and C. Sibilia, Rev. Sci. Instrum. 64, 1576 (1993).

${ }^{5}$ J. A. Batista, A. M. Mansanares, E. C. da Silva, C. C. Vaz, and L. C. M. Miranda, J. Appl. Phys. 88, 5079 (2000).
${ }^{6}$ V. E. Gusev, Acustica 82, S37 (1996).

${ }^{7}$ O. Matsuda and O. B. Wright (unpublished).

${ }^{8}$ Electronic Handbook of Optical Constants of Solids, edited by E. D. Palik and G. Ghosh (SciVision, Academic, New York, 2000).

${ }^{9}$ Thermophysical Properties of Matter, edited by Y. S. Touloukian, R. We. Powell, C. Y. Ho, and P. G. Klemens (Plenum, New York, 1970), Vol. 2.

${ }^{10}$ Thermophysical Properties of Matter, edited by Y. S. Touloukian and E. H. Buyco (Plenum, New York, 1970), Vol. 5.

${ }^{11}$ American Institute of Physics Handbook, 3rd ed., edited by D. E. Gray (McGraw-Hill, New York, 1972).

${ }^{12}$ Thermophysical Properties of Matter, edited by Y. S. Touloukian (Plenum, New York, 1970), Vol. 13

${ }^{13}$ Equation (6) is derived from the thermal diffusion equation assuming, as in the one-dimensional case, surface absorption in the substrate.

${ }^{14}$ The particular type of normalization of the curves in Fig. 1 to a common point at $10 \mathrm{kHz}$ implies that the curves for the one- and three-dimensional theories are not coincident at high frequencies in the plots in Fig. 1.

${ }^{15}$ The $1 / f^{1 / 2}$ dependence is only exact in the limit in which the probe optical absorption depth is much smaller than the thermal diffusion length. Likewise, the phase lag only becomes exactly equal to $45^{\circ}$ in the same limit. This limit is effectively reached in the present experiments because for silicon the probe optical absorption depth is $2.4 \mu \mathrm{m}$, whereas the smallest thermal diffusion length is $17 \mu \mathrm{m}$ in the present chopping frequency range (see Table I)

${ }^{16}$ M. Bertolotti, G. Liakhou, R. Li Voti, F. Michelotti, and C. Sibilia, J. Appl. Phys. 74, 7078 (1993).

${ }^{17}$ I. S. Gradshteyn and I. M. Ryzhik, Table of Integrals, Series, and Products (Academic, New York, 1994). 